a four-year-old synthetic-biology company in Boston, Massachusetts, that develops 'made-to-order microbes' to churn out marketable chemicals. Founder Jason Kelly says that the company plans to charge customers on the basis of how much they use the microbes. For accurate billing and theft protection, Gingko needs to control that use, so it is developing what Kelly calls a 'gene-guard' technology: a genetic tweak that makes production of the desired chemical dependent on a proprietary additive, supplied by Ginkgo, in its fermentation medium. The approach could even be used in nanotechnology, by making engineered nanobots that are dependent on a proprietary raw material.

In a strange twist of fate, terminator technology has begun to look more appealing to environmentalists. Organic farmers want ways to keep genetically engineered crops from contaminating their fields, and food-safety groups are concerned about contamination of food crops with products from a new generation of crops engineered to produce chemicals or pharmaceuticals. By ensuring that genetically modified plants survive for only one planting, "that technology would have alleviated a lot of environmental concerns", says Holman.

Approaches dependent on switching will take considerable research, however; companies reported technical challenges with the first generation of terminator techniques. Patents owned by Monsanto required the insertion of three different genes into the plant genome. Monsanto says it is currently not researching the techniques, and other companies are hoping that they will not have to. "Perhaps these technologies could provide new ways to protect investments," says Brett Lund, former head of intellectual property for the biofuels group of Syngenta, an agri-giant headquartered in Basel, Switzerland. "But the easiest and best way is through our patent system."

\section{TERMINATOR, THE SEQUEL}

As the US Supreme Court hears a controversial gene-patent case, biotechnology companies are once again considering ways to limit naturally self-replicating technologies.

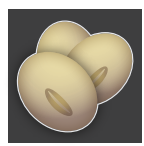

\section{SEEDS}

'Terminator' technology produces sterile seeds.

Status: Politically toxic.

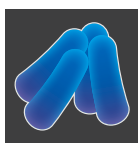

\section{SYNTHETIC BIOLOGY}

'Gene guard' needs proprietary supplement in growth medium. Status: Under development.

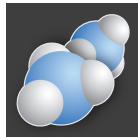

NANOTECHNOLOGY

Requirement for proprietary

supplement to 'grow'.

Status: Hypothetical.

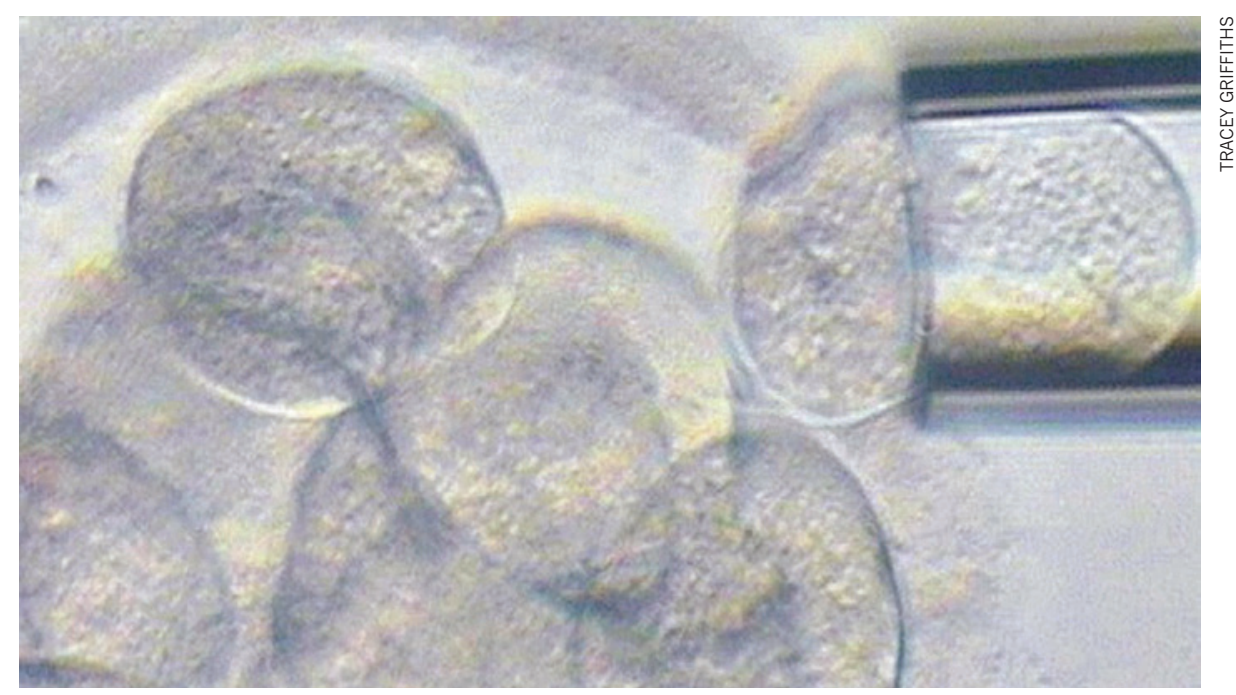

Single-cell genomics is allowing fertility clinics to screen embryos for abnormalities more cheaply.

GENOMICS

\title{
Gene sequencing leaves the laboratory
}

\section{Maturing technology speeds medical diagnoses.}

\section{BY ERIKA CHECK HAYDEN}

$\mathrm{T}$ The steep fall in the cost of sequencing a genome has, for the moment, slowed. Yet researchers attending this year's Advances in Genome Biology and Technology (AGBT) meeting in Marco Island, Florida, on 20-23 February are not complaining. At a cost as low as US\$5,000-10,000 per human genome, sequencing has become cheap and reliable enough that researchers are not waiting for the next sequencing machine to perfect new applications in medicine.

Two recent beneficiaries: embryo screening after in vitro fertilization (IVF) and metagenomic medicine, which entails sequencing many different microbes en masse and then teasing out individual genomes to diagnose which ones are helping or harming human health. "The field is maturing," says geneticist Jay Shendure at the University of Washington in Seattle.

At last year's AGBT meeting, Oxford Nanopore, based in Oxford, UK, electrified researchers by unveiling details of a machine that could sequence DNA at unprecedentedly low cost and high speed - but that machine has not yet arrived on the market. This year, no splashy new technologies are expected. Instead, the HiSeq machine, made by Illumina, based in San Diego, California, has tightened its grip on the top end of the market (see 'Reasonably priced genomes').
Borrowing a metaphor from computer-circuit development, quantitative biologist Michael Schatz at Cold Spring Harbor Laboratory in New York says that after many "tick" years of dramatic technological innovation, the field is now in a "tock" period as researchers fine-tune the technology and expand it into applications.

One application depends on single-cell genomics, a focus of this year's meeting. To sequence a genome from a single cell, the DNA has to be amplified, a process that can introduce errors into the sequence. But new amplification techniques now allow accurate sequences to be obtained from individual cells, including the precious cells extracted from human embryos.

At the meeting, Dagan Wells at the University of Oxford, will describe how the technique can be used to select which of the embryos created by IVF has the best chance of developing into a healthy baby. Fertility experts already screen embryos for genetic abnormalities by extracting a single cell from each eight-cell embryo and running a variety of tests to detect chromosomal abnormalities, but this can cost around a thousand dollars per IVF cycle.

Instead, Wells extracts DNA from the sampled cell, amplifies it and sequences a part of the genome using the Personal Genome Machine, a fast sequencer made by Ion Torrent in Guilford, Connecticut, a subsidiary 
of Life Technologies in Carlsbad, California. Within a day, Wells can detect duplications or deletions of entire chromosomes - the abnormalities seen most often early in development. Several women are now pregnant with embryos selected using this technique, and a larger clinical trial is planned for later this year. Wells says that the low cost - as much as 50\% less than current methods - should open up testing to more women.

Other researchers are refining existing technologies to increase the average length of the DNA strand that can be 'read' from the sequencer. The devices that dominate the field sequence short fragments of DNA, no more than a few hundred base pairs, and overlook some portions of the genome. Longer reads are needed, for example, to distinguish between closely related microorganisms - an important requirement for researchers studying whether overabundance of some microbial species in the gut or vagina make patients more susceptible to problems ranging from diarrhoea to premature birth.

Late last year, Illumina bought Moleculo, a company based in San Francisco, California, whose technology extends reads by changing the way that DNA is prepared for sequencing and by applying new analytical software to the readout. These tricks can coax reads thousands

\section{REASONABLY PRICED GENOMES}

Although no reports of big innovations in DNA sequencing are expected at a major conference this week the current cost and capabilities of the technology now make medical applications worthwhile.

\begin{tabular}{l|l|l|l|} 
Name & Machine cost & Read length (bases) & Cost per megabase \\
Illumina MiSeq & US $\$ 125,000$ & 500 & $14-70$ cents \\
Illumina HiSeq & US $\$ 690,000$ & 300 & $4-5$ cents \\
PacBio RS & US $\$ 695,000$ & 4,575 & $\$ 2-17$ \\
Ion Torrent PGM & US $\$ 49,000$ & 400 & 60 cents- $\$ 5$ \\
Ion Torrent Proton & US $\$ 224,000$ & 200 & $1-9$ cents
\end{tabular}

of bases long out of Illumina machines - an order of magnitude greater than previously possible. The machines produced by Pacific Biosciences in Menlo Park, California, also achieve read lengths that routinely reach into the thousands of bases, thanks to an engineered polymerase. Customers say that the company seems to have overcome early problems with reliability.

In market share, Pacific Biosciences still has a long way to go to catch Illumina or Life Technologies, which this month said that it expects sales of the Ion Torrent sequencing system to boost revenue by up to $5 \%$ in 2013 . Corporate takeovers are a topic of fevered speculation in the sequencing world. Rumours that Life Technologies' sequencing business may be acquired sent its shares soaring to their highest price ever. And Illumina recently fended off a hostile takeover bid by Swiss company Roche in Basel, which formally dropped its offer a month ago. But the fundamental landscape has not changed much.

Any reshuffling of the major players will have to wait for the field's next 'tick' cycle, whether it takes the form of Life Technologies' Ion Proton machine - which is just hitting labs and which the company says will eventually deliver a $\$ 1,000$ genome in a day - or Oxford Nanopore's GridION and MinION machines, which scientists still haven't seen. In the meantime, says Shendure, "this year's meeting will be less glitz and more science, and that's great".

\section{Dark-matter hunt gets deep}

\section{China launches world's deepest particle-physics experiment - but it joins a crowded field.}

\section{BY EUGENIE SAMUEL REICH}

$\mathrm{M}$ ore than 1,000 metres underground, physicists have set traps of liquid xenon to catch their prey: hypothetical particles of dark matter that might very rarely interact with ordinary matter as they drift through Earth. With construction costs on the order of US $\$ 10$ million each, such experiments are a relatively cheap way to work out the composition of $85 \%$ of the matter in the Universe. But does the world really need four of them?

Ongoing experiments in Italy, the United States and Japan are now being joined by a fourth in China, called PandaX (see 'Dark and deep'). Installed in the deepest laboratory in the world, 2,500 metres under the marble mountain of JinPing in Sichuan province, PandaX will this year begin monitoring 120 kilograms of xenon. The team hopes to scale the tank up to 1 tonne by 2016 , which would mean that the experiment had developed more quickly than any other dark-matter search. "We want to demonstrate

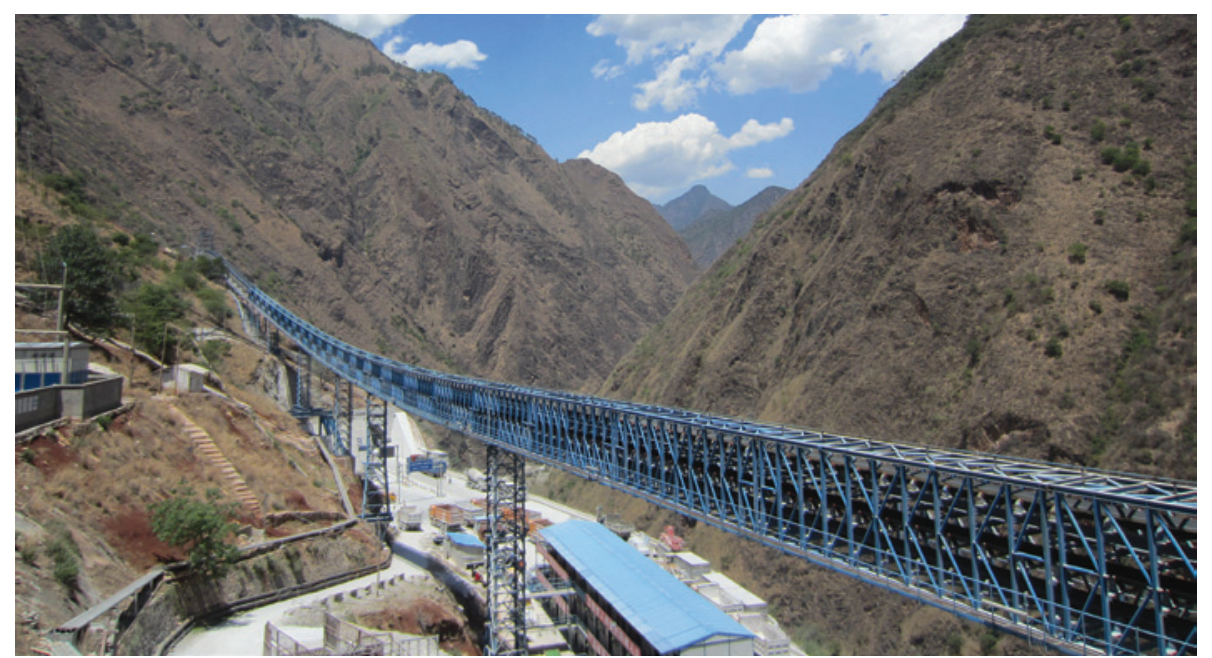

A conveyor belt removes rock from JinPing laboratory, a 2,500-metre-deep dark-matter experiment site.

that world-class research in dark matter is possible in China," says Xiangdong Ji, a physicist at Shanghai Jiao Tong University in China and a spokesman for PandaX.
Dark-matter researchers in the West are excited by the ambition of the project, but some question the duplication of effort. "Spending all our money on different direct-detection 